\title{
The role of alteplase in preventing relapsing and repeat peritonitis in peritoneal dialysis
}

\author{
Joana M. Martins ${ }^{1}$, Joel Ferreira ${ }^{2}$, Ricardo A. Macau ${ }^{3}$, Fernando T. Costa ${ }^{1}$, Aura Ramos ${ }^{1}$ \\ ${ }^{1}$ Nephrology Department, Hospital Garcia de Orta, Portugal \\ 2 Diaverum Group, Portugal \\ ${ }^{3}$ Nephrology Department, Centro Hospitalar de Leiria
}

\section{ABSTRACT}

Introduction: Peritonitis is a common complication in peritoneal dialysis, playing a weighty role in patient morbidity and a major cause of transfer to hemodialysis Relapsing or repeat episodes are associated with the development of a biofilm-trapping bacteria, and catheter removal is recommended in these cases. Since October of 2013 our peritoneal dialysis unit has developed a protocol that adds a fibrinolytic agent to an intraperitoneal antibiotic, aiming to reduce the number of relapsing/repeat episodes.

Objective: To demonstrate the efficacy and safety of associating a fibrinolytic agent to intraperitoneal antibiotics in the prevention of relapsing or repeat peritonitis.

Methodology: Observational study comparing the number of relapsing/repeat peritonitis events between two groups during a four-year period: a historic one, treated with intraperitoneal antibiotics only (control group) and one group that received an association of intraperitoneal antibiotics with alteplase (alteplase group). Secondary and fungi peritonitis were excluded from this study.

Results: During the study period, a total of 103 peritonitis episodes were registered (control group: 61 episodes; alteplase group: 42 episodes) corresponding to 17 relapsing/repeat events, with statistical significance between groups (control group: 15 episodes (24.6\%); alteplase group: 2 episodes $(4,7 \%) ; p=0.008)$. There was no difference in demographic characteristics or the presence of exit site infection between the groups. The microorganisms most frequently involved in relapsing/repeat episodes were S.epidermidis and E.coli. Regarding the outcomes of the treatment, no patient in the alteplase group had to undergo catheter removal due to relapsing/repeat episodes of peritonitis. There were no adverse events following alteplase administration. Three deaths due to peritonitis-related sepsis were registered.

Conclusion: Although relapsing/repeat peritonitis are still an important cause of catheter removal, this study demonstrates the potential benefit of fibrinolytic association with intraperitoneal antibiotics in preventing these events and potentially reducing modality drop-out.

Keywords: peritoneal dialysis, peritonitis, tissue plasminogen activator

\section{INTRODUCTION}

Peritonitis is a common and major complication of peritoneal dialysis (PD), leading to structural and functional alterations in the peritoneal membrane, and thereby to membrane failure and conversion to hemodialysis (HD) ${ }^{1}$. Peritonitis is diagnosed when at least two of the following criteria are present: (1) clinical features consistent with peritonitis, e.g. abdominal pain and/or cloudy dialysis effluent; (2) dialysis effluent white cell count $>100 / \mu \mathrm{L}$ or $>0.1 \times 10^{9} / \mathrm{L}$ (after a dwell time of at least 2 hours), with $>50 \%$ polymorphonuclear; and (3) positive dialysis effluent culture ${ }^{1}$. Antibiotic treatment should be started once diagnosis is established, with intraperitoneal (IP) antibiotics the first choice.

Relapsing peritonitis is defined by an episode that occurs within four weeks of completion of therapy for a prior episode of peritonitis caused by the same organism or one sterile episode. Repeat peritonitis is an episode that occurs more than 4 weeks after completion of therapy for a prior episode of peritonitis caused by the same organism ${ }^{1}$. It is thought that there is a colonization of the peritoneal dialysis catheter by bacteria that create a biofilm, leading to refractory or relapsing peritonitis ${ }^{2-4}$. These peritonitis episodes are associated with a lower rate of cure, more frequent ultrafiltration problems, and higher rate of technique failure ${ }^{1,5}$. The use of fibrinolytic agents has been considered for refractory or relapsing peritonitis. The rationale for the use of fibrinolytic agents is that bacteria are trapped in fibrin within the peritoneum and/or the Tenckhoff catheter, protecting them from antibiotic action ${ }^{4}$. Prior studies by electron-microscopy have found that viable organisms were trapped within extracellular material (glycocalyx and/or fibrin) associated with the catheter ${ }^{6}$. If fibrin is lysed, bacteria are released and become vulnerable to antibiotic therapy by increasing biofilm permeability and the antibiotic penetration. However, trials have failed to show any benefit of IP urokinase in the treatment of refractory peritonitis ${ }^{2-4}$. The International Society of Peritoneal Dialysis (ISPD) still recommends catheter removal in refractory and relapsing peritonitis ${ }^{1}$.

Alteplase is a thrombolytic agent, a tissue plasminogen activator (tPA) produced by recombinant DNA technology, converting plasminogen to plasmin. Alteplase binds to fibrin in a thrombus and converts the entrapped plasminogen to plasmin, initiating local fibrinolysis ${ }^{7}$. 
Its use is recommended in obstructed HD and PD catheter, restoring the patency of the lumen 8,9 . The most serious adverse events reported after treatment with alteplase were bleeding from gastrointestinal tract ${ }^{7}$.

Before October 2013, in our department, peritonitis episodes were treated only with intraperitoneal antibiotics. Since then, a protocol has been established that adds a fibrinolytic agent (alteplase) to the IP antibiotics.

The aim of this study was to evaluate the efficacy and safety of an intraperitoneal fibrinolytic agent in the prevention of repeat or relapsing peritonitis rate.

\section{MATERIAL AND METHODS}

Observational study from October 2013 to December of 2017, comparing the number of relapsing/repeat peritonitis since the alteplase protocol was initiated - alteplase group (AG), with historical data for the same time period (2009 to October of 2013) - control group (CG).

Diagnosis of peritonitis was established according to ISPD guidelines ${ }^{1}$ and IP empiric antibiotherapy was initiated with ceftazidime and cefazolin, in both groups. Antibiotic adjustments were made as soon as the bacterial agent was isolated, and the length of treatment was established following ISPD guidelines ${ }^{1}$. Fungi and secondary peritonitis were excluded from our study.

Patients in the AG received IP alteplase on day $3(5 \mathrm{mg})$ and day $10(7.5 \mathrm{mg})$ of every peritonitis episode. After infusion of dialysis solution with antibiotics in peritoneal cavity, alteplase was diluted in 5 to $7.5 \mathrm{cc}$ of distilled water and was instilled into the Tenckhoff catheter, which was clamped for 2 hours. After a 2-hours permanence, the catheter was washed with 10 to $20 \mathrm{cc}$ of normal saline solution.

Statistic data were evaluated with SPSS 22 using Chi square for categorical data and Student's T-test for continuous variables.

\section{RESULTS}

During the evaluation period, 103 peritonitis episodes were considered [42 (40.78\%) in the AG and $61(59.22 \%)$ in the CG] in a total of 51 patients ( 22 from AG and 29 from CG) (Table I).

There was no significant difference in the demographic characteristics between the two groups. The time on PD was about the same, as was the prevalence of diabetes in both groups [AG: 11 (50\%); CG: $9(31 \%), p=0.17)$. As for other risk factors for peritonitis and exit site infections, there was no statistical difference between the groups (AG: 3 (7.1\%), CG: 2 (3.3\%), $p=0.18$ ) (Table I).

Most patients in both groups were on automated peritoneal dialysis (APD) (Table I). In the control group, most of the relapsing/repeat episodes occurred on APD [AG: $0(0 \%)$ vs. CG=13 (86.7\%)], while in $A G$, both relapsing events occurred on continuous ambulatory peritoneal dialysis (CAPD) (Table I).

Most peritonitis episodes were caused by Gram positive bacteria, the most common in both groups being S.epidermidis [AG: 15 peritonitis (35.7\%); CG:14 peritonitis (22.9\%)] (Table II). E. coli was the most frequent Gram negative agent in the control group; in the alteplase group there was no predominance of any Gram negative bacteria agent (Table II).

Two (AG) and 15 (CG) episodes of relapsing/repeat peritonitis were recorded (Table I), showing an important decrease in the number of relapsing/repeat episodes of peritonitis since the beginning of the use of alteplase [AG: $4.7 \%$ vs. CG $24.6 \%, p=0.008$ ).

In the alteplase group, the relapsing/repeat episodes were caused by $S$. epidermidis, while in the control group, other agents were responsible for relapsing/repeat peritonitis episodes (S. epidermidis, E. coli, S. aureus, Corynebacterium spp, St viridans, and S. haemolyticus) (Table III). In the control group, one of the episodes was a sterile peritonitis (Table III).

Regarding outcomes, there was no difference between the groups in peritonitis-related hospitalization (AG: 12 (28.6\%), CG:

\section{Table I}

Number of peritonitis, relapsing/repeat episodes and patients' demographic characteristics. Numbers are given as absolute or mean \pm standard deviation.

\begin{tabular}{|c|c|c|c|c|c|}
\hline & \multicolumn{2}{|c|}{ Alteplase Group } & \multicolumn{2}{|c|}{ Control Group } & \multirow{2}{*}{$\frac{p \text { value }}{-}$} \\
\hline Peritonitis episodes (n) & 42 & $40.78 \%$ & 61 & $59.22 \%$ & \\
\hline Relapsing/repeating episodes (n) & 2 & $4.7 \%$ & 15 & $24.6 \%$ & 0.008 \\
\hline Patients with peritonitis ( $\mathrm{n}$ ) & 22 & $43.1 \%$ & 29 & $56.9 \%$ & - \\
\hline Patients on APD (n) & 13 & $59.1 \%$ & 21 & $72.4 \%$ & 0.32 \\
\hline Relapsing peritonitis in APD (n) & 0 & $0 \%$ & 13 & $86.7 \%$ & - \\
\hline Men (n) & 16 & $72.7 \%$ & 22 & $75.9 \%$ & 0.79 \\
\hline Age (in years) & $65.78 \pm 13.79$ & & $57.6 \pm 17.33$ & & 0.14 \\
\hline Diabetes (n) & 11 & $50 \%$ & 9 & $31 \%$ & 0.17 \\
\hline Time in peritoneal dialysis (in months) & $25.02 \pm 17.08$ & - & $23.12 \pm 17.98$ & - & 0.31 \\
\hline Individuals with prior peritonitis episodes (n) & 10 & $45.4 \%$ & 17 & $58.6 \%$ & 0.3 \\
\hline Exit site infection association (n) & 3 & $7.1 \%$ & 2 & $3.3 \%$ & 0.37 \\
\hline
\end{tabular}




\section{Table II}

Distribution of causative agents of peritonitis

\begin{tabular}{l|c|c} 
& Alteplase Group & Control Group \\
\hline Peritonitis episodes (n) & 42 & 61 \\
Microorganisms & & \\
$\quad$ S. Epidermidis & 15 & 14 \\
S. Aureus & 1 & 9 \\
Corynebacterium spp. & 1 & 6 \\
E. coli & 1 & 5 \\
St. viridans & 2 & 8 \\
S. haemolyticus & 1 & 6 \\
S. pasteuri & 1 & 0 \\
S. hominis & 1 & 0 \\
St. salivarius & 1 & 0 \\
St. GrB-Bhaemolyticus & 2 & 0 \\
Neisseria spp & 2 & 0 \\
$\quad$ Enterococcus faecalis & 0 & 2 \\
Others & 7 & 4 \\
Sterile & 7 & 7
\end{tabular}

\section{Table III}

Microorganisms causing relapsing/repeat peritonitis

\begin{tabular}{l|c|c|c} 
& Alteplase Group & Control Group & $p$ value \\
\hline Relapsing/repeating episodes (n) & 2 & 15 & 0.008 \\
Microorganisms & & & \\
$\quad$ S. Epidermidis & 2 & 3 & - \\
S. Aureus & 0 & 2 & - \\
Corynebacterium spp. & 0 & 2 & - \\
E. coli & 0 & 4 & - \\
St. viridans & 0 & 1 & - \\
$\quad$ S. haemolyticus & 0 & 2 & - \\
Sterile & 0 & 1 & -
\end{tabular}

$19(31.1 \%) p=0.8)$ (Table IV). The number of patients whose PD catheter was removed was higher in the control group $(n=8)$ than in the alteplase group $(n=5)$, but with no statistical significance (Table IV). Regarding catheter removal, in the control group, three were due to relapsing/repeat peritonitis episodes while in the alteplase group no patient had catheter removal for that reason (Table IV). However, these results had no statistical significance
(AG: $0(0 \%), C G: 3$ catheter removal due to relapsing/repeat episodes $(4.9 \%), p=0.15)$

Three deaths were registered, two in the control group and one in the alteplase group. In both groups the cause of death was septic shock related to peritonitis (Table IV).

No adverse event from the administration of alteplase was registered.

\section{DISCUSSION}

Peritoneal dialysis is an important therapeutic option for the management of end stage kidney disease and peritonitis is the major cause of morbidity related to this technique. For this reason, prevention and treatment of this complication is an extremely important issue. Historically there was a reduction in the rate of peritonitis caused by Gram positive organisms after the introduction of the Y-set and the double bag disconnection system ${ }^{10}$.

The use of fibrinolytic agents in peritonitis has been discussed and its use is recommended for de-obstruction of the peritoneal catheter ${ }^{9}$. However, the evidence that fibrinolytic agents improve the outcomes of relapsing peritonitis is poor, failing to show any benefit of IP urokinase in the treatment of refractory peritonitis ${ }^{2-4}$. However, there are some case reports and single-center uncontrolled series using urokinase which showed that the addition of this fibrinolytic to IP antibiotics in patients with relapsing peritonitis due to coagulase-negative staphylococci could eradicate the organism ${ }^{11}$. In 2004, in a single-center study ${ }^{12}$, tPA was administrated to five patients with relapsing peritonitis due to $S$. epidermidis and in three of them there was no recurrence ${ }^{12}$. More recently, a pre-clinical research study demonstrated that alteplase was stable in standard dialysate solutions and in the presence of antibiotics, and safe when given intraperitoneally in a mouse model, with no evidence of local or systemic toxicity ${ }^{13}$. This same study concluded that alteplase may have a role in the management of PD peritonitis ${ }^{13}$.

Before October 2013, our unit used only IP antibiotics for the treatment of peritonitis. On October 2013 we started adding alteplase, a fibrinolytic agent, to reduce the incidence of relapsing or repeat peritonitis. This agent was administered on the third day, filling the catheter lumen, and on the tenth day in a larger amount to enter the peritoneal

\section{Table IV}

Clinical outcomes of peritonitis episodes. Numbers are given as absolute

\begin{tabular}{|c|c|c|c|c|c|}
\hline & \multicolumn{2}{|c|}{ Alteplase Group } & \multicolumn{2}{|c|}{ Control Group } & \multirow{2}{*}{$\frac{p \text { value }}{-}$} \\
\hline Number of peritonitis ( $n$ ) & 42 & & 61 & & \\
\hline Catheter removal (n) & 5 & $11.9 \%$ & 8 & $13.1 \%$ & 0.85 \\
\hline Relapsing/repeating episodes (n) & 0 & $0 \%$ & 3 & $4.9 \%$ & 0.15 \\
\hline Catheter malfunction ( $\mathrm{n}$ ) & 1 & $2.4 \%$ & 1 & $1.6 \%$ & 0.79 \\
\hline Modality transfer (n) & 4 & $9.5 \%$ & 4 & $6.6 \%$ & 0.58 \\
\hline Hospitalization (n) & 12 & $28.6 \%$ & 19 & $31.1 \%$ & 0.8 \\
\hline Deaths (n) & 1 & & 2 & & - \\
\hline
\end{tabular}


cavity, and destroy all adherences. Similarly to that reported in previous studies ${ }^{12}$, we also registered no adverse event related to alteplase administration.

Analyzing the results showed an important reduction in the number of relapsing peritonitis since we started using alteplase [AG:2 (4.7\%) vs CG: $15(24.6 \%), p=0.008)$ so our belief is that the use of fibrinolytic agent enhanced the antibiotics' penetration and action.

Most of the peritonitis episodes are due to technique errors, so they are expected to occur more often at the beginning of the technique ${ }^{10}$. Nevertheless, in both groups the time from starting PD until the peritonitis episodes was on average over 20 months. These data can reinforce the importance of a periodical revaluation of the patients' technique.

According to the literature, the rates of peritonitis on APD and on CAPD are not generally different ${ }^{14}$. In our study, most of our patients are on APD, with a high prevalence of peritonitis in both groups [AG: 13/22 (59.1\%); CG: 21/29 (72.4\%); $p=0.32$ ] (Table I).

As in the work by Zelenitsky et al (2000) ${ }^{15}$, in our population, peritonitis was caused most commonly by Staphylococcus epidermidis and E.coli was the most prevalent of the Gram negative agents ${ }^{15}$. In our study, these agents were also the ones associated with more relapsing episodes (Table III).

Although there was no statistical difference between groups, there was a reduction in the number of hospitalizations and catheter removal in the AG (Table IV).

Comparing catheter removal between groups, three of the eight removals in the CG were due to relapsing/repeat peritonitis (assuming catheter colonization), while none of the five removals in the AG was for that reason (AG: 0\%, CG:4.9\%, $\mathrm{p}=0.15$ ) (Table IV).

Regarding the number of hospitalizations [AG: 12 (28.6\%), CG: 19 (31.1\%), $p=0.87$ ], most of them lasted only a day or two to reinforce patients' education and ensure the safety of the technique (Table IV).

Three deaths were registered in total, two in the control group and one in the alteplase group. All of them were due to septic shock due to peritonitis. The agents involved were Klebsiella pneumoniae, Corynebacterium spp. and E.coli multi drug resistant (MDR), respectively.

Despite the small study size, we found a significant reduction of relapsing/repeat peritonitis episodes with the introduction of the alteplase protocol. We acknowledge some limitations: being a retrospective study, comparing the results with historical controls, it does not take into consideration other developments in clinical practice (e.g. more frequent reinforcement of PD technique). However, subsequent relapsing/repeat peritonitis events are a hard end-point that are not open to interpretation and were significantly lower after the use of fibrinolytic agents.

\section{CONCLUSION}

Although ISPD still recommends catheter removal for relapsing peritonitis ${ }^{1}$, and despite the small study size, our study suggests that fibrinolytic agents may play a role in preventing relapsing or repeat peritonitis when associated with IP antibiotics, thus potentially reducing the need to remove the catheter and drop-out from PD program. The role of fibrinolytics in increasing biofilm permeability may facilitate antibiotics' penetration, reducing the number of relapsing/repeat episodes. It is important to record that no adverse event from the administration of fibrinolytics was registered; thus alteplase administration seems to be safe in PD.

Disclosure of potential conflicts of interest: none declared.

\section{References}

1. Philip Kam-Tao Li,1 Cheuk Chun Szeto,1 Beth Piraino, et al. "ISPD peritonitis recommendations: 2016 update on prevention and treatment", PDI, July 2016.

2. Tong MK, Leung KT, Siu YP, Lee KF, Lee HK, Yung CY, et al. Use of intraperitoneal urokinase for resistant bacterial peritonitis in continuous ambulatory peritoneal dialysis. J Nephrol 2005; 18:204-8.

3. Gadallah MF, Tamayo A, Sandborn M, Ramdeen G, Moles K. Role of intraperitoneal urokinase in acute peritonitis and prevention of catheter loss in peritoneal dialysis patients. Adv Perit Dial 2000; $16: 233-6$

4. Innes A, Burden RP, Finch RG, Morgan AG. Treatment of resistant peritonitis in continuous ambulatory peritoneal dialysis with intraperitoneal urokinase: a double-blind clinical trial. Nephrol Dial Transplant 1994; 9:797-9. 341.

5. Szeto CC, Kwan BC, Chow KM, Law MC, et al. Recurrent and relapsing peritonitis: causative organisms and response to treatment. Am J Kidney Dis 2009; 54:702-10.

6. Read RR, Eberwein P, Dasgupta MK et al. Peritonitis in peritoneal dialysis: bacterial colonisation by biofilm spread along the catheter surface. Kidney Int 1989; 35: 614-21.

7. Genentech, prescribing information for Cathflo ${ }^{\circledR}$ Activase ${ }^{\oplus}$ [alteplase], 2001.

8. Adeera Levin, Michael Rocco, Garabed Eknoyan. 2006 Updates Clinical Practice Guidelines and Recommendations. KDOQI National Kidney Foundation CPRs for Vascular Access, 2006: 320-7.

9. Paolo Strippoli, Domenico Pilolli, Giuliano Mingrone, Arcangelo Dimaggio et al. A Hemostasis Study in CAPD Patients During Fibrinolytic Intraperitoneal Therapy with Urokinase (UK). Adv Perit Dial 5:97-9, 1989.

10. Monteon F, et al. Prevention of peritonitis with disconnect systems in CAPD: a randomizes control trial. The Mexican Nephrology Collaborative study Group. Kidney Int. 1998: 54: 2123-38.

11. Pickering SJ, Fleming SJ, Bowley JA, Sissons P, Oppenheim BA, et al. (1989) Urokinase: a treatment for relapsing peritonitis due to coagulase-negative staphylococci. Nephrol Dial Transplant. 4: 62-5.

12. Zorzanello MM, Fleming WJ, Prowant BE. Use of tissue plasminogen activator in peritoneal dialysis catheters: a literature review and one center's experience. Nephrol Nurs. J. 2004, Sep- Oct; 31 (5):534-7.

13. McGuire AL, Bennett SC, Lansley SM, Popowicz ND, Varano della Vergiliana JF, Wong D, et al. (2015) Preclinical Assessment of Adjunctive tPA and DNase for Peritoneal Dialysis Associated Peritonitis. PLOS ONE 10(3): e0119238 doi:10.1371/journal pone.0119238.

14. John T Daugirdas, Peter G Blake, Todd S.Ing. Handbook f dialysis, 5th edition, Lippincott Williams \& Wilkins, 2015: 490-512.

15. Sheryl Zelenitsky, PharmD, Linda Barns, BSc, lan Findlay, BSc, et al. Analysis of Microbiological Trends in Peritoneal Dialysis-Related Peritonitis From 1991 to 1998. American Journal of Kidney Diseases, Vol 36, No 5 (November), 2000: pp 1009-13.

\section{Correspondence to:}

Joana M. Martins, MD

Nephrology Department, Hospital Garcia de Orta, Portugal

E-mail: joana.marquesmartins@gmail.com 